\title{
反相气相色谱法探究镁铁水滑石及其改性材料的表面性质
}

\author{
徐金芳，郡蒙蒙，倪哲明，肖雪春 \\ (浙江工业大学 化学工程学院, 杭州 310014)
}

摘 要: 采用反相气相色谱法(IGC)探究司班 20(Span-20)、吐温-80(Tween-80)、曲拉通 X-100(Triton X-100)分别与 十二烷基苯磺酸钠(SDBS)复合改性镁铁水滑石材料(即 SDBS/Span-LDHs、SDBS/Tween-LDHs、SDBS/Triton-LDHs) 的表面性质, 通过表面吸附自由能 $\left(-\Delta G^{0}\right)$ 、表面能色散组分 $\left(\gamma_{\mathrm{s}}{ }^{\mathrm{d}}\right)$ 及酸碱作用自由能 $\left(-\Delta G^{\mathrm{sp}}\right)$ 这三个参数探讨材料改性 前后的变化。结果表明, 水滑石经改性后的 $-\Delta G^{0}$ 值均比未改性材料的值小, 其中 SDBS/Triton-LDHs 的 $-\Delta G^{0}$ 值最小, 说明 SDBS/Triton-LDHs 材料的稳定性最好，且水滑石材料改性后碱性减弱，其中 SDBS/Triton-LDHs 材料的碱性最 弱。此外, 各类水滑石的 $\gamma_{\mathrm{s}}^{\mathrm{d}}$ 值均随着温度的升高而减小, 因此, 在制备聚合物/水滑石类材料时, 可提高温度来改善 其与聚合物的相容性。

关 键 词: 阴-非离子表面活性剂; 水滑石; 反相气相色谱法; 表面性质

中图分类号: O614 文献标识码: A

\section{Surface Properties of Magnesium-iron Hydrotalcite and Its Modified Products by Inverse Gas Chromatography}

\author{
XU Jin-Fang, SHAO Meng-Meng, NI Zhe-Ming, XIAO Xue-Chun \\ (College of Chemical Engineering, Zhejiang University of Technology, Hangzhou 310014, China)
}

\begin{abstract}
The magnesium-iron hydrotalcite was modified by three nonionic surfactants (Span-20, Tween-80, Triton X-100) combined with sodium dodecyl benzene sulfonate (SDBS), respectively, and surface characterizations of modified hydrotalcites were investigated using inverse gas chromatography (IGC). The free energy of adsorption $\left(-\Delta G^{0}\right)$, dispersive component of the surface energy $\left(\gamma_{\mathrm{s}}{ }^{\mathrm{d}}\right)$ and acid-basic action free energy $\left(-\Delta G^{\mathrm{sp}}\right)$ were determined to explore the changes between $\mathrm{MgFe}-\mathrm{LDHs}$ and modified hydrotalcites. The results show that the $-\Delta G^{0}$ of the modified hydrotalcites are lower than that of MgFe-LDHs, among which the $-\Delta G^{0}$ of the SDBS/Triton-LDHs is the lowest, indicating the best stability. The alkalinity of modifed hydrotalcites is weakened and the alkalinity of SDBS/Triton-LDHs is the weakest. In addition, the $\gamma_{\mathrm{s}}{ }^{\mathrm{d}}$ decreases with temperature increase. Therefore, the compatibility with polymer can be improved by increasing the temperature in preparation of polymer/hydrotalcite materials.
\end{abstract}

Key words: anionic-nonionic surfactant; hydrotalcite; inverse gas chromatography; surface characterization

水滑石(Layered double hydroxides, LDHs)是一类 新型的无机功能材料, 其化学组成为: $\left[\mathrm{M}^{2+}{ }_{1-x} \mathrm{M}^{3+}{ }_{x}\right.$ $\left.(\mathrm{OH})_{2}\right]^{x+}\left(\mathrm{A}^{n-}\right)_{x / n} \cdot m \mathrm{H}_{2} \mathrm{O}^{[1]}$ 。该双金属氢氧化物由于其
特殊的层板结构被广泛应用于吸附、催化、离子交 换等方面 ${ }^{[2-4]}$ 。但 LDHs 的组成和结构中含有大量的 结构水、吸附水，颗粒表面又存在大量的非架桥羟 
基，使得其粒子结合在一起易发生团聚。因而，为增 强水滑石粒子在高分子基体中的分散性, 提高其催 化效率, 拓展其应用领域, 需要对 LDHs 进行表面 改性，克服其团聚特性。

表面活性剂可以对水滑石表面进行改性, 在水 滑石粒子表面产生空间阻斥力, 从而减弱或屏蔽粒 子的团聚现象, 达到表面改性目的。表面活性剂有 阴离子型、阳离子型、非离子型以及混合型等。阴非离子表面活性剂是一种混合型表面活性剂 ${ }^{[5]}$, 不 仅保持了各表面活性剂的优点, 还存在协同效应使 其具有高于单一表面活性剂的性能。 $\mathrm{Li}$ 等 ${ }^{[6]}$ 利用耗 散颗粒动力学模拟了 SDBS 与 Triton X-100 在油水 界面的作用, 发现表面活性剂以团簇的形式存在, 同时两者产生协同作用，降低界面张力。Zhang 等 ${ }^{[7]}$ 发现有机粘土经阴-非离子表面活性剂复合改性后, 有利于对五氯苯酚的吸附, 且其体系的稳定性也有 所增强。本课题组曾报道 SDBS 和 Tween-80 复合改 性镁铝水滑石的改性机理 ${ }^{[8]}$, 但不同非离子表面活 性剂复合改性水滑石的效果、变化规律及其机理则 尚未见报道。因此, 为水滑石改性剂的选择提供较 好的依据，为更好地开拓水滑石的应用领域，有必 要对不同非离子表面活性剂与阴离子表面活性剂复 合改性水滑石后表面性质的变化加以研究。

反相气相色谱法(IGC) 是以待测材料作为固定 相, 研究材料表面性质的分析方法, 已广泛应用于 表征聚合物、离子液体、氧化物等各类材料 ${ }^{[9-11]}$ 。 Karakehya 等 ${ }^{[12]}$ 就曾用 IGC 法研究过 PVC 不饱和 聚酯中加入不同含量蒙脱石后的复合材料表面酸碱 性质。本研究利用 IGC 法探讨了三种非离子表面活性 剂吐温-80(Tween-80)、曲拉通 X-100(Triton X-100)、 司班 20(Span-20)分别与十二烷基苯磺酸钠(SDBS) 复合, 对镁铁水滑石表面性质的影响规律, 研究改 性后水滑石的表面吸附自由能 $\left(-\Delta G^{0}\right)$ 、表面能色散 组分 $\left(\gamma_{\mathrm{s}}{ }^{\mathrm{d}}\right)$ 、表面酸碱性等参数的变化, 探讨不同非离 子表面活性剂与 SDBS 复合改性水滑石表面性质的 变化。

\section{1 实验方法}

\section{1 样品的制备}

通过双滴定共沉淀法 ${ }^{[13]}$ 制备出镁铁水滑石 $(\mathrm{MgFe}-$ $\mathrm{LDHs})$ : 称取一定量的 $\mathrm{MgCl}_{2} \cdot 6 \mathrm{H}_{2} \mathrm{O}$ 及 $\mathrm{Fe}\left(\mathrm{NO}_{3}\right)_{3} \cdot 9 \mathrm{H}_{2} \mathrm{O}$ (镁铁物质的量之比 $2: 1$ ) 溶于去离子水中, 配成 溶液 $\mathrm{A}$; 称取一定量的 $\mathrm{Na}_{2} \mathrm{CO}_{3}$ 及 $\mathrm{NaOH}$ 溶于去离子 水中, 配成溶液 $\mathrm{B}$ 。在强烈的磁力摚拌下将溶液 $\mathrm{A}$ 、
溶液 $\mathrm{B}$ 分别滴加到装有一定量去离子水的三口烧瓶 中, 并控制 $\mathrm{pH}$ 值在 9 10 之间, 滴完之后继续搅拌 $1 \mathrm{~h} 。 65{ }^{\circ} \mathrm{C}$ 下晶化 $18 \mathrm{~h}$, 经抽滤、洗涤后得到滤饼, 将 该滤饼放入 $65{ }^{\circ} \mathrm{C}$ 的烘箱内干燥后, 研磨至粉末样 品, 记为 $\mathrm{MgFe}-\mathrm{LDHs}$ 。

取未经干燥过的水滑石滤饼, 并加入一定量的 去离子水, 使其稀释成质量分数为 $10 \%$ 的浆液。将 SDBS(分析纯)与 Triton X-100(化学纯)按一定比例 混合, 即为阴-非离子表面活性剂, 取 $5 \mathrm{wt} \%$ 的阴-非 离子表面活性剂于浆液中, $80{ }^{\circ} \mathrm{C}$ 的油浴中搅拌反应 $30 \mathrm{~min}$, 浆液经抽滤、洗涤、干燥后, 研磨至粉末, 记为 SDBS/Triton-LDHs。

依次将 Triton X-100 换成 Tween-80(化学纯)、 Span-20(化学纯), 按照上述方法制备出一系列阴非离子表面活性剂复合改性镁铁水滑石的样品, 分 别记为 SDBS/Tween-LDHs、SDBS/Span-LDHs。

\section{2 试剂与表征}

选用正成烷, 正己烷, 正庚烷, 正辛烷, 二氯 甲烷, 四氯化碳, 苯作为探针分子, 所用试剂均为 分析纯。

采用德国布鲁克光谱仪器公司生产的 Bruker Vector 22 型傅里叶变换红外光谱仪分析样品的结 构。采用荷兰帕纳科公司生产的 $\chi^{\prime}$ Pert PRO 型 X 射 线粉末衍射仪 $(\mathrm{Cu}$ 靶, $\mathrm{K} \alpha$ 射线, $\lambda=0.1542 \mathrm{~nm}, 40 \mathrm{kV} /$ $40 \mathrm{~mA}$ ) 测定样品的晶体结构, 扫描范围 $2 \theta=5^{\circ} \sim 70^{\circ}$ 。

采用天美仪器公司生产的 GC 7890( II) 型气相 色谱仪研究样品的表面性质, 配有热导池检测器 (TCD), 选用氮气作为载气。气化室和检测器温度均 设置为 $170{ }^{\circ} \mathrm{C}$, 柱温范围为 $130 \sim 160{ }^{\circ} \mathrm{C}$, 变化间隔 为 $10{ }^{\circ} \mathrm{C}$ 。

\section{3 色谱柱的制备}

所选用的色谱柱为内径 $0.2 \mathrm{~cm}$, 长度 $15 \mathrm{~cm}$ 的 不锈钢柱, 依次经丙酮、去离子水清洗后, 自然晾干, 分别将已制备的一系列阴-非离子表面活性剂复合 改性后的水滑石样品均匀填入色谱柱中, 记录装柱 前与装柱后的色谱柱质量, 求出固定相的质量。将 制备好的色谱柱于 $160{ }^{\circ} \mathrm{C}$ 下通氮气老化 $1 \mathrm{~h}$ 。

\section{2 结果与讨论}

\subsection{XRD 及 FTIR 分析}

图 1 为镁铁水滑石及不同阴-非离子表面活性 剂复合改性后水滑石的 XRD 图谱。从图 1 可以看 出, 四种材料均在低 $2 \theta$ 角处出现了水滑石的 $(003)$ 、 (006)、(009)特征晶面峰。通过布拉格公式计算出 


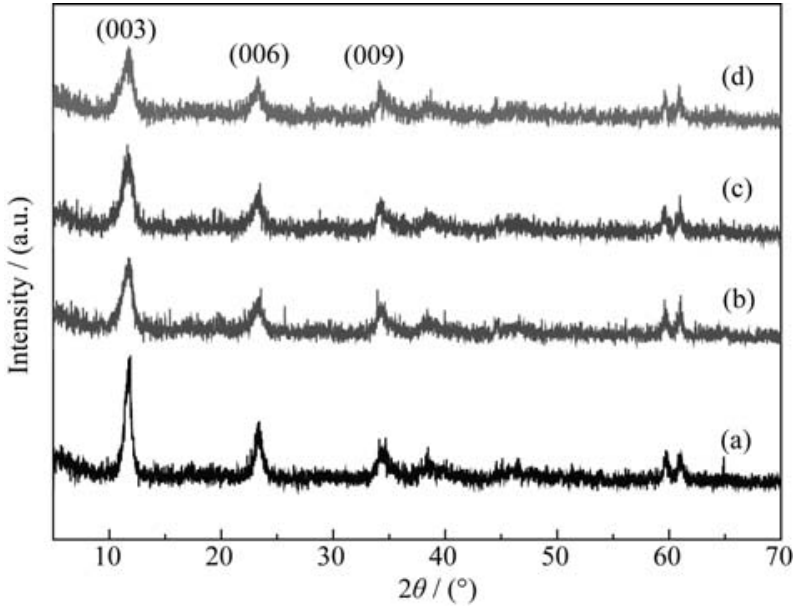

图 1 镁铁水滑石及其改性后水滑石的 XRD 谱图

Fig. 1 XRD patterns of MgFe-LDHs and modified LDHs (a) MgFe-LDHs; (b) SDBS/Triton-LDHs; (c) SDBS/Tween-LDHs; (d) SDBS/Span-LDHs

MgFe-LDHs、SDBS/Triton-LDHs、SDBS/Tween-LDHs、 SDBS/Span-LDHs 这四种材料的层间距依次为: $0.758 、 0.761 、 0.760 、 0.760 \mathrm{~nm}$ 。经改性后的 $\mathrm{MgFe}-$ LDHs 层间距基本不变, 表明表面活性剂几乎未进 入镁铁水滑石的层间。

图 2 为镁铁水滑石及不同阴-非离子表面活性 剂复合改性后水滑石的 FTIR 谱图, 从图中可以看 出四种不同的水滑石材料均在 $581 \mathrm{~cm}^{-1} 、 744 \mathrm{~cm}^{-1}$ 处出现了层板金属键的吸收峰, $1359 \mathrm{~cm}^{-1}$ 处出现了 层间 $\mathrm{CO}_{3}{ }^{2-}$ 的振动吸收峰, $3443 \mathrm{~cm}^{-1}$ 处出现了层间水 和层板羟基的伸缩振动峰, 表明 $\mathrm{MgFe}-\mathrm{LDHs}$ 已成 功合成，且水滑石经改性之后，层间阴离子仍存在。 SDBS/Triton-LDHs、SDBS/Tween-LDHs 和 SDBS/ Span-LDHs 曲线在 $2856 \mathrm{~cm}^{-1} 、 2926 \mathrm{~cm}^{-1}$ 处出现了

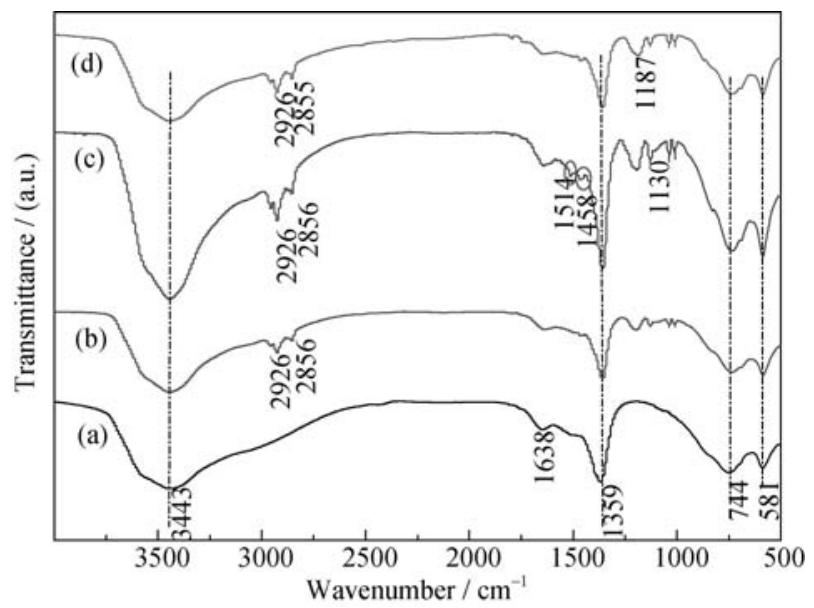

图 2 镁铁水滑石及其改性后的水滑石的 FTIR 图谱

Fig. 2 FTIR spectra of MgFe-LDHs and modified LDHs (a) MgFe-LDHs; (b) SDBS/Triton-LDHs; (c) SDBS/Tween-LDHs; (d) SDBS/Span-LDHs
$-\mathrm{CH}_{3} 、-\mathrm{CH}_{2}-$ 的伸缩振动峰, 在 $1187 \mathrm{~cm}^{-1}$ 处出现了 $\mathrm{SDBS}$ 中的 $-\mathrm{SO}_{3}{ }^{-}$的伸缩振动峰, 在 $1130 \mathrm{~cm}^{-1}$ 附近出 现了 $-\mathrm{C}-\mathrm{O}-\mathrm{C}-$ 的反对称伸缩振动峰。而 SDBS/ Tween-LDHs 在 1458、1514 $\mathrm{cm}^{-1}$ 出现了苯环的特征 吸收峰。这些结果显示, SDBS/Triton、SDBS/ Tween、 $\mathrm{SDBS} / \mathrm{Span}$ 成功改性了镁铁水滑石。结合 XRD 分析 结果，提示四种改性水滑石中的表面活性剂几乎未 进入镁铁水滑石层间，而是以表面改性为主。

\section{2 反相气相色谱分析}

\subsection{1 改性后水滑石的表面吸附自由能}

本实验条件下，探针分子进入色谱柱后被无限 稀释，吸附遵循亨利定律，吸附自由能可由如下公 式求得 ${ }^{[14-16]}$.

$$
\Delta G^{0}=-\mathrm{R} T \ln \left(\frac{V_{\mathrm{N}} P_{\mathrm{o}}}{\pi_{\mathrm{o}} m S}\right)
$$

式中, $\mathrm{R}$ 为理想气体常数; $T$ 为色谱柱的温度; $P_{\mathrm{o}}$ 为 各探针分子在标准状态下的饱和蒸汽压; $\pi_{\mathrm{o}}$ 为固体 表面二维铺张压; $m$ 为色谱柱中固定相(水滑石及改 性后的水滑石材料)的质量; $S$ 为固定相的比表面积; $V_{\mathrm{N}}$ 为探针分子在固定相中的保留体积, 可通过探针 分子在色谱柱中的保留时间测得：

$$
\begin{gathered}
V_{\mathrm{N}}=\left(t_{\mathrm{R}}-t_{\mathrm{m}}\right) \cdot F_{\mathrm{a}} \cdot \frac{T}{T_{\mathrm{a}}} \cdot J \\
J=\frac{3}{2}\left[\frac{\left(p_{\mathrm{i}} / p_{\mathrm{o}}\right)^{2}-1}{\left(p_{\mathrm{i}} / p_{\mathrm{o}}\right)^{3}-1}\right]
\end{gathered}
$$

式中, $t_{\mathrm{R}} 、 t_{\mathrm{m}}$ 分别为探针分子在色谱柱中的保留时 间、死时间; $F_{\mathrm{a}}$ 为载气流速; $T 、 T_{\mathrm{a}}$ 分别为柱温、室 温; $J$ 为 James-Martin 压力校正因子; $p_{\mathrm{i}} 、 p_{\mathrm{o}}$ 分别为进 口压力、出口压力。

将各值代入公式(1)、(2)、(3)中, 求出柱温 $150{ }^{\circ} \mathrm{C}$ 下正构烷烃在固定相表面的吸附自由能, 具体数值 见表 1 。

横向比较表 1 中的数据, 发现该四种水滑石材 料表面的吸附自由能值 $\left(-\Delta G^{0}\right)$ 均随着正构烷烃 $\left(\mathrm{C}_{5}-\mathrm{C}_{8}\right)$ 中碳链长度的增加而减小, 表明正构烷烃随 着碳链长度的增加, 更难以进入水滑石材料的结构 中, 并且更难以被水滑石材料吸附。

纵向比较表 1 中的数据, 发现 $\mathrm{MgFe}-\mathrm{LDHs}$ 经阴非离子表面活性剂复合改性之后, 其吸附自由能的 值 $\left(-\Delta G^{0}\right)$ 均减小，表明阴-非离子表面活性剂改性 $\mathrm{MgFe}-\mathrm{LDHs}$ 后，有利于提高水滑石的稳定性，这可 能是由于阴-非离子表面活性剂中非离子表面活性 剂优先形成胶团，阴离子表面活性剂逐渐进入已形 成的胶团中，最终形成的混合胶团 ${ }^{[17]}$ 掩盖了层板上 的活性羟基基团，从而提高其稳定性。另外，三种 
表 1 柱温 $150{ }^{\circ} \mathrm{C}$ 下探针分子在各类水滑石材料表面的吸附自由能

Table 1 Surface free energy for the adsorption of probes on LDHs at $150{ }^{\circ} \mathrm{C}$

\begin{tabular}{ccccc}
\hline \multirow{2}{*}{ System } & \multicolumn{3}{c}{$-\Delta G^{0} /\left(\mathrm{kJ} \cdot \mathrm{mol}^{-1}\right)$} \\
\cline { 2 - 5 } & n-pentane & n-hexane & n-heptane & n-octane \\
\hline MgFe-LDHs & 15.23 & 14.38 & 14.24 & 13.06 \\
SDBS/Span-LDHs & 12.89 & 9.68 & 7.15 & 5.67 \\
SDBS/Tween-LDHs & 12.01 & 8.93 & 6.46 & 4.61 \\
SDBS/Triton-LDHs & 11.82 & 8.43 & 5.94 & 4.31 \\
\hline
\end{tabular}

改性材料中 SDBS/Triton-LDHs 体系的吸附自由能 值最小。一方面是由于 Trition X-100 的分子量较大, 所形成的胶团粒径较大 ${ }^{[18]}$, 掩盖更多的活性羟基基 团 ${ }^{[19-20]}$, 使其稳定性较好; 另一方面, SDBS 中的 $-\mathrm{SO}_{3}{ }^{-}$与 Trition X-100 的聚氧乙烯基团存在较强的 离子-偶极相互作用 ${ }^{[21-22]}$, 阴-非离子表面活性剂得 到充分的聚合，最终使 SDBS/Triton-LDHs 在四种材 料中的稳定性最好。

\subsection{2 改性后水滑石的表面能色散组分}

材料表面的性质通常以活性、表面积、酸碱性 等来表征 ${ }^{[23]}$, 活性的大小直接影响材料在聚合物中 的分散性 ${ }^{[24]}$, 且表面能色散组分 $\left(\gamma_{\mathrm{s}}^{\mathrm{d}}\right)$ 可较好地表示 活性的大小，衡量固体表面极化探针分子的能力, 具体计算公式如下 ${ }^{[25]}$ :
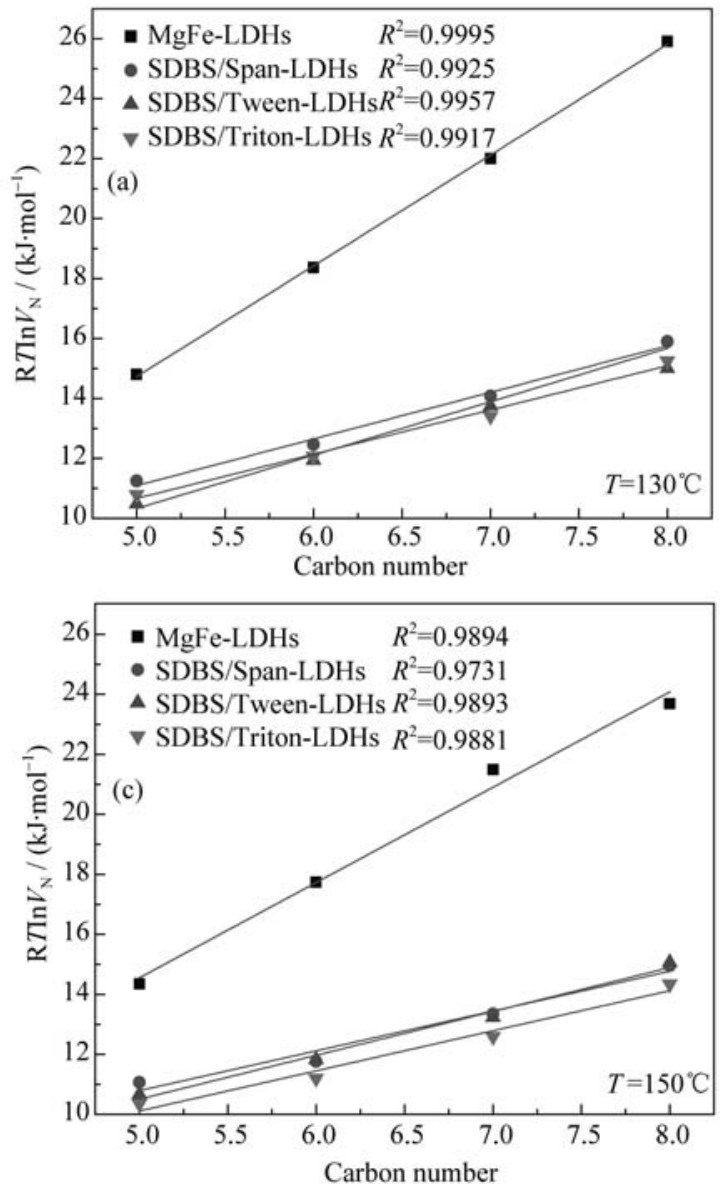

$$
\gamma_{\mathrm{s}}^{\mathrm{d}}=\frac{1}{\gamma_{\mathrm{CH}_{2}}} \cdot\left(\frac{-\Delta G_{\mathrm{CH}_{2}}}{2 N \alpha_{\mathrm{CH}_{2}}}\right)^{2}
$$

其中, $\gamma_{\mathrm{CH}_{2}}$ 为仅由 $-\mathrm{CH}_{2}$ 基团组成固体的表面能; $N$ 为 Avogadro 常数; $\alpha_{\mathrm{CH}_{2}}$ 为一个亚甲基基团的横截面 积 $\left(\alpha_{\mathrm{CH}_{2}}=0.06 \mathrm{~nm}^{2}\right) ; \Delta G_{\mathrm{CH}_{2}}$ 为吸附自由能的增量, 在 一定温度下，正构烷烃分子 $\left(\mathrm{C}_{5} \sim \mathrm{C}_{8}\right)$ 在固定相表面的 $\mathrm{R} T \operatorname{In} V_{\mathrm{N}}$ 与其碳原子数成线性关系，直线的斜率为吸 附自由能的增量 $\left(\Delta G_{\mathrm{CH}_{2}}\right)$, 如图 3 所示。

通过图 3 中的线性相关系数 $R^{2}$ 发现该四种水 滑石材料的 $\mathrm{R} T \mathrm{In} V_{\mathrm{N}}$ 与正构烷烃 $\left(\mathrm{C}_{5} \sim \mathrm{C}_{8}\right)$ 含碳原子数 均呈现出良好的线性关系，说明通过图中的斜率 求出的 $\Delta G_{\mathrm{CH}_{2}}$ 值具有较好的可靠性, 结合公式(4), 求出该四类水滑石材料在不同温度下的 $\gamma_{\mathrm{s}}^{\mathrm{d}}$, 如表 2 所示。
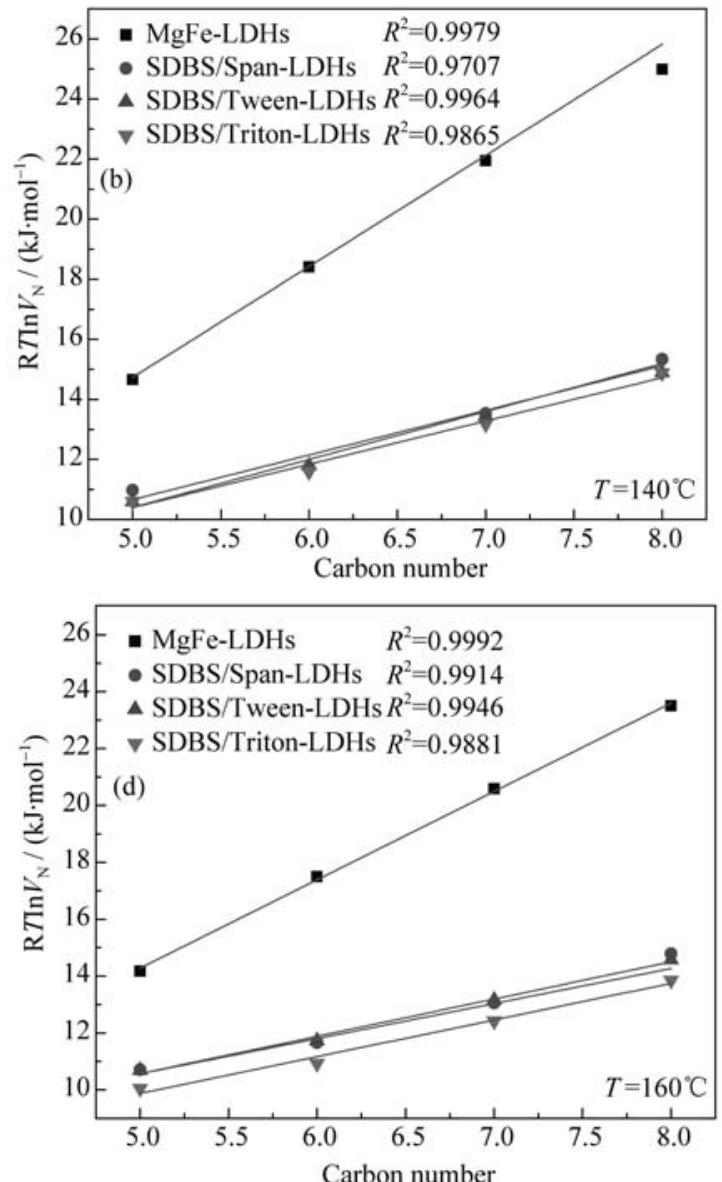

图 3 不同温度下各类水滑石表面的 $\mathrm{R} T \operatorname{In} V_{\mathrm{N}}$ 随正构烷烃所含碳原子数的变化

Fig. 3 Plot of RTIn $V_{\mathrm{N}}$ values versus carbon number at different temperatures 
表 2 水滑石在不同温度下的表面能色散组分 $\gamma_{\mathrm{s}}{ }^{\mathrm{d}}$

Table $2 \gamma_{s}^{d}$ values of LDHs at different temperature

\begin{tabular}{ccccc}
\hline System & \multicolumn{3}{c}{$\gamma_{\mathrm{s}}^{\mathrm{d}} /\left(\mathrm{mJ} \cdot \mathrm{m}^{-2}\right)$} \\
\cline { 2 - 5 } & $130{ }^{\circ} \mathrm{C}$ & $140{ }^{\circ} \mathrm{C}$ & $150{ }^{\circ} \mathrm{C}$ & $160{ }^{\circ} \mathrm{C}$ \\
\hline MgFe-LDHs & 195.00 & 177.55 & 158.81 & 158.13 \\
SDBS/Span-LDHs & 34.50 & 32.93 & 31.87 & 30.58 \\
SDBS/Tween-LDHs & 33.67 & 31.58 & 29.68 & 28.14 \\
SDBS/Triton-LDHs & 31.02 & 30.99 & 28.13 & 27.27 \\
\hline
\end{tabular}

纵向比较表 2 中的数据, 发现 MgFe-LDHs 经不 同阴-非离子表面活性剂改性后, 其 $\gamma_{\mathrm{s}}^{\mathrm{d}}$ 值均有不同程 度的减小。这可能是由于 $\mathrm{MgFe}-\mathrm{LDHs}$ 经阴-非离子表 面活性剂改性后，层板上的活性羟基逐渐被表面活性 剂掩盖, 从而减弱极化探针的能力, 使 $\gamma_{\mathrm{s}}^{\mathrm{d}}$ 值减小。

横向比较表 2 中的数据, 随着温度的升高, 该 四类水滑石材料的 $\gamma_{\mathrm{s}}{ }^{\mathrm{d}}$ 值均逐渐减小, 这是由于随着 温度的升高，探针分子与该四类水滑石类材料间的 距离逐渐增大, 而 $\gamma_{\mathrm{s}}{ }^{\mathrm{d}}$ 值随着探针分子与水滑石类材 料间距离的增大而逐渐减小。此外, $\gamma_{\mathrm{s}}{ }^{\mathrm{d}}$ 值越小, 该材 料与聚合物间的相容性越好。因此, 在制备水滑石/ 聚合物材料时, 可通过升高温度来改善水滑石与聚 合物间的相容性。

\subsection{3 改性后水滑石的表面酸碱性}

表面酸碱性是一种重要的固体材料表面性质, 通过材料表面的酸碱性的测定, 可从理论上指导聚 合物填料的选择。酸碱作用自由能 $\left(-\Delta G^{\mathrm{sp}}\right)$ 是一个可 以定性地表征材料表面酸碱性质的重要参数。本研 究以 $\mathrm{R} T \operatorname{In} V_{\mathrm{N}}$ 对探针分子蒸汽压的对数值 $\left(\lg P_{\mathrm{o}}\right)$ 作图 的方法求出 $-\Delta G^{\mathrm{sp}}$ 值, 具体数值见表 3 。

从表 3 中的数据可见该四类水滑石材料与碱性 探针分子 $\left(\mathrm{C}_{6} \mathrm{H}_{6}\right)$ 之间具有较大的 $-\Delta G^{\mathrm{sp}}$ 值, 表明材料 表面酸性较强; 另外, 该四类材料与酸性探针分子 $\left(\mathrm{CCl}_{4} 、 \mathrm{CH}_{2} \mathrm{Cl}_{2}\right)$ 之间仍存在酸碱作用力, 表明材料表 面具有一定的碱性位, 这与水滑石表面具有羟基有 关, 但其 $-\Delta G^{\mathrm{sp}}$ 值较小。这些结果说明材料表面的碱 性较弱, 该四类材料均属于两性偏酸材料。

表 3 柱温 $140{ }^{\circ} \mathrm{C}$ 下不同探针分子在各类水滑石材料 表面的 $-\Delta G^{\mathrm{s}}$ 值

Table $3-\Delta G^{\text {sp }}$ values of probles on LDHs at $140{ }^{\circ} \mathrm{C}$

\begin{tabular}{cccc}
\hline \multirow{2}{*}{ System } & \multicolumn{3}{c}{$-\Delta G^{\mathrm{sp}} /\left(\mathrm{kJ} \cdot \mathrm{mol}^{-1}\right)$} \\
\cline { 2 - 4 } & $\mathrm{CCl}_{4}$ & $\mathrm{CH}_{2} \mathrm{Cl}_{2}$ & $\mathrm{C}_{6} \mathrm{H}_{6}$ \\
\hline MgFe-LDHs & -1.14 & 2.44 & 3.37 \\
SDBS/Span-LDHs & 0.81 & 1.95 & 5.82 \\
SDBS/Tween-LDHs & 0.47 & 1.75 & 3.74 \\
SDBS/Triton-LDHs & 0.32 & 1.35 & 1.59 \\
\hline
\end{tabular}

纵向比较表 3 中的数据, 阴-非离子表面活性剂 复合改性 $\mathrm{MgFe}-\mathrm{LDHs}$ 后, 其与酸性探针分子 $\left(\mathrm{CCl}_{4}\right.$ 、 $\mathrm{CH}_{2} \mathrm{Cl}_{2}$ ) 间的 $-\Delta G^{\mathrm{sp}}$ 值均减小, 这是由于 $\mathrm{MgFe}-\mathrm{LDHs}$ 表面含有大量的羟基，使其表面呈现出一定的碱性， 经表面改性后，表面的羟基以不同程度被表面活性 剂覆盖, 使得改性后水滑石表面的碱性减弱, 其中 SDBS/Triton-LDHs 材料表面羟基的覆盖率最大，因 此 SDBS/Triton-LDHs 的碱性最弱, 且从表 3 中的数 据可见其酸性也减弱。这意味着 SDBS/Triton-LDHs 在做聚合物填料时, 对酸碱聚合物均适合, 而 $\mathrm{MgFe}$ LDHs、SDBS/Span-LDHs 及 SDBS/Tween-LDHs 这 三种材料具有较强的酸性, 更适合做酸性聚合物 的填料。

\section{3 结论}

本研究将一系列非离子表面活性剂分别与阴离 子表面活性剂复合用于改性美铁水滑石, 研究得出 如下结论:

1) 通过对 MgFe-LDHs、SDBS/Triton-LDHs、 SDBS/Tween-LDHs、SDBS/Span-LDHs 四种材料的 表面吸附自由能 $\left(-\Delta G^{0}\right)$ 计算, 发现 MgFe-LDHs 经阴 -非离子表面活性剂改性后, 其稳定性均有所提高, 且其中 SDBS/Triton-LDHs 的稳定性最好。

2) 通过表面能色散组分 $\left(\gamma_{s}{ }^{d}\right)$ 计算, 发现四种材 料的 $\gamma_{\mathrm{s}}{ }^{\mathrm{d}}$ 值均随着温度的升高而逐渐减小，从而提高 材料与聚合物的相容性，在制备水滑石/聚合物材料 时，可以通过升高温度来改善水滑石与聚合物间的 相容性。

3) 通过酸碱作用自由能 $\left(-\Delta G^{\mathrm{sp}}\right)$ 的计算, 发现该 四种水滑石材料表面均具有酸性位和碱性位, $\mathrm{MgFe}$ LDHs 经阴-非离子表面活性剂改性后碱性减弱, 其中 SDBS/Triton-LDHs 的碱性最弱, 且该材料的酸性也减 弱, 用于做酸性与碱性聚合物填料时均适合。

\section{参考文献:}

[1] TSUJIMURA A, UCHIDA M, OKUWAKI A. Synthesis and sul- 
fate ion-exchange properties of a hydrotalcite-like compound intercalated by chloride ions. Journal of Hazardous Materials, 2007, 143(1/2): 582-586.

[2] NOVILLO C, GUAYA D, AVENDANO A A P, et al. Evaluation of phosphate removal capacity of $\mathrm{Mg} / \mathrm{Al}$ layered double hydroxides from aqueous solutions. Fuel, 2014, 138: 72-79.

[3] TAN L C, WANG Y L, LIU Q, et al. Enhanced adsorption of uranium (VI) using a three-dimensional layered double hydroxide/ graphene hybrid material. Chemical Engineering Journal, 2015, 259: 752-760.

[4] FAN G L, LI F, EVANS D G, et al. Catalytic applications of layered double hydroxides: recent advances and perspectives. Chemical Society Reviews, 2014, 43(20): 7040-7066.

[5] 肖进新, 赵振果. 表面活性剂应用原理. 北京: 化学工业出版 社, 2003: 49-51.

[6] LI Y, HE X J, CAO X L, et al. Molecular behavior and synergistic effects between sodium dodecylbenzene sulfonate and Triton X-100 at oil/water interface. Journal of Colloid and Interface Science, 2007, 307(1): 215-220.

[7] ZHANG Y X, LONG Y Y, ZHANG Y C, et al. Effect of a mixed anionic-nonionic surfactant adsorption on bentonite structure and on distribution of pentachlorophenol. Applied Clay Science, 2012, 69: 93-98.

[8] XU JIN-FANG, SHI WEI, NI ZHE-MING, et al. Surface characteriization for anionic-nonionic surfactant modified layered double hydroxides. Chinese Journal of Inorganic Chemistry, 2014, 30(5): 977-983.

[9] PAPADOPOULOU S K, PANAYIOTOU C. Thermodynamic characterization of poly(1,1,1,3,3,3-hexafluorois opropyl methacrylate) by inverse gas chromatography. Journal of Chromatography A, 2012, 1229: 230-236.

[10] CORDEIRO N, ORNELAS M, ASHORI A, et al. Investigation on the surface properties of chemically modified natural fibers using inverse gas chromatography. Carbohydrate Polymers, 2012, 87(4): 2367-2375.

[11] MOISE J C, MUTELET F, JAUBERT J N, et al. Activity coefficients at infinite dilution of organic compounds in four new imidazolium-based lonic liquids. Journal of Chemical and Engineering Data, 2011, 56(7): 3106-3114.

[12] KARAKEHYA N, BILGIC C. Surface characterisation of montmorillonite/PVC nanocomposites by inverse gas chromatogra-phy. International Journal of Adhesion and Adhesives, 2014, 51: 140-147.

[13] WEI M, XU X Y, HE J, et al. Preparation and thermal decomposition studies of L-tyrosine intercalated $\mathrm{MgAl}, \mathrm{NiAl}$ and $\mathrm{ZnAl}$ layered double hydroxides. Journal of Physics and Chemistry of
Solids, 2006, 67(7): 1469-1476.

[14] RUCKRIEM M, INAYAT A, ENKE D, et al. Inverse gas chromatography for determining the dispersive surface energy of porous silica. Colloids and Surfaces A-Physicochemical and Engineering Aspects, 2010, 357(1/2/3): 21-26.

[15] FU XIAO-WEI, NI ZHE-MING, LIU JIAO. Surface characterization of copper-aluminum-magnesium hydrotalcites investigated by inverse gas chromatography. Acta Chimica Sinica, 2012, 79(8): 968-972.

[16] WANG W J, HUA Q, SHA Y F, et al. Surface properties of solid materials measured by modified inverse gas chromatography. $\mathrm{Ta}$ lanta, 2013,112: 69-72.

[17] YANG CHUN-SHENG, CUI XIAO-HONG, JIANG YAN, et al. Mixed micelles of sodium dodecyl sulfate and triton X-100 in aqueous solution studied by ${ }^{1} \mathrm{H}$ NMR. Chinese Journal of Magnetic Resonance, 2009, 26(4): 466-475.

[18] LIU JIN-YAN, HAN WAI-HUI, ZHANG YAN, et al. Studies on the conductivity of mixed reverse micelles of anionic and non-ionic surfactanta. Acta Physico-Chimica Sinica, 2010, 26(6): $1552-1556$.

[19] XU SHENG, ZENG HONG-YAN, ZHAO CE, et al. Surface modification of Mg-Al hydrotalcite flame-retardant. Journal of The Chinese Ceramic Society, 2013, 41(4): 527-533.

[20] Hernandez M, Fenandez L, Borras C, et al. Characterization of surfactant/hydrotalcite-like clay/glassy carbon modified electrodes: oxidation of phenol. Analytica Chimica Acta, 2007, 597: 245-256.

[21] LI HAI-CHAO, LIU JIN-YAN, ZHANG YING-CHUN, et al. Interaction of sodium dodecyl benzene sulfonate and Triton X-100 studied by intrinsiic fluorescence. Chinese Journal of Applied Chemistry, 2011, 28(1): 113-117.

[22] DEB N, SHANNIGRAHI M, BAGCHI S. Use of fluorescence probes for studying kamlet-taft solvatochromic parametes of micellar system formed by binary mixture of sodium dodecyl sulfate and Triton-X 100. Journal of Physical Chemistry B, 2008, 112(10): $2868-2873$.

[23] VOELKEL A, STRZEMIECKA B, ADAMSKA K, et al. Inverse gas chromatography as a source of physiochemical data. Journal of Chromatography A, 2009, 1216(10): 1551-1566.

[24] QU XIAO-HONG, MA XIAO-YAN, CHEN FANG, et al. Correlation of the structure of clay filled polyurethane with surface properties. Acta Materiae Compositae sinica, 2007, 3(24): 13-17.

[25] MA X Y, QU X H, ZHANG Q L, et al. Analysis of interfacial action of rectorite/thermoplastic polyurethane nanocomposites by inverse gas chromatography and molecular simulation. Polymer, 2008, 49: 3590-3600. 